\title{
Location and Extent of A1, A2 Release and Its Impact on Tendon Subluxation and Bowstringing-A Cadaveric Study
}

\author{
Laxminarayan Bhandari ${ }^{1,2} \quad$ Alireza Hamidian Jahromi ${ }^{2} \quad$ Aden Gunnar Miller ${ }^{1} \quad$ Huey Tien ${ }^{1}$ \\ ${ }^{1}$ Christine M. Kleinert Institute of Hand and Microsurgery, Louisville, \\ Kentucky, United States \\ 2Department of Plastic and Reconstructive Surgery, University of \\ Tennessee, Memphis, Tennessee, United States \\ Address for correspondence Laxminarayan Bhandari, MD, Christine \\ M. Kleinert Institute of Hand and Microsurgery, Louisville, KY 40202, \\ United States (e-mail: lax321@gmail.com).
}

Indian J Plast Surg 2019;52:349-354

\begin{abstract}
Surgical treatment of trigger finger involves release of A1 pulley. Some authors have theorized that the loss of A1 pulley can lead to ulnar subluxation of flexor tendons, which can be prevented by release of A 1 pulley radially, even in a nonrheumatoid hand. However, there is no evidence in literature to either support or oppose this hypothesis. Occasionally, difficulty is encountered to precisely identify where $A 1$ ends and $A 2$ begins. While incomplete release of $A 1$ can cause relapse of triggering, release of substantial $A 2$ can cause bowstringing. Knowledge of the safe limit of concomitant $A 2$ release is beneficial. The study was conducted in 12 cadaver upper extremity specimens. A1 pulleys of 48 fingers were divided at the radial ( 24 fingers) or ulnar ( 24 fingers) attachment. A $20 \mathrm{lb}$ traction force was applied on the flexor tendons. Any subluxation or bowstringing was noted. The experiment was repeated following serial release of the A2-initially $25 \%$, followed by $50 \%$ and $100 \%$. No bowstringing or subluxation was noted when $A 1$

Keywords

- trigger finger

- A1 pulley

- A2 pulley

- tendon subluxation

- complication pulley was opened, either by radial or ulnar incision. The same was true for $A 1+25 \%$ $A 2$ release. When $A 1+50 \% A 2$ pulley were released, bowstringing was observed in $3 / 48$ fingers. When $A 1+100 \%$ of the $A 2$ pulley were released, bowstringing occurred in all cases. The location of incision for release of the A1 pulley has no effect on bowstringing or tendon subluxation. Release of additional $25 \%$ of the A2 pulley can be performed safely, which corresponds to the level of palmar digital crease.
\end{abstract}

\section{Introduction}

Trigger finger is a common condition of the hand that occurs when the annular A1 pulley becomes abnormally stiff and thickened, and along with inflammation of the flexor tendons eventually inhibits the tendon glide through the pulley. Conservative treatment involves a local steroid injection into the flexor tendon sheath, but this method is not always successful to resolve the condition. Surgical treatment is very effective and involves release of the A1 pulley. The biomechanical effects of A1 pulley release have been studied by various authors and, although controversial, are believed to be insignificant by most authors. ${ }^{1}$
However, some authors have suggested that loss of A1 pulley function can lead to metacarpophalangeal volar subluxation, bowstringing, and ulnar deviation, with subsequent effects on hand function and its active range of motion..$^{2-4}$ Such effects were proposed to occur in both rheumatoid and nonrheumatoid hands. ${ }^{2,3} \mathrm{~A} 1$ pulley could potentially provide additional stability to the metacarpophalangeal joint and assists to prevent subluxation and ulnar drift of fingers. ${ }^{3}$ Lin et al, in a cadaveric study, evaluated the mechanical properties of the fibro-osseous pulleys in the hand by stressing the pulleys by a custom-made loading device. Their study concluded the A1 pulley to be the third strongest pulley of the upper extremities after the A2 and A4 pulleys. ${ }^{5}$ Some authors
DOI https://doi.org/

10.1055/s-0039-3402705

ISSN 0970-0358.
(C2019 Association of Plastic Surgeons of India
License terms

(요 (1) $\Theta \circledast$ 
have gone further and advocated a routine stepwise reconstruction of the A1 pulley following the trigger finger release in an attempt to counteract these potential problems. ${ }^{4}$

Progressive ulnar deviation of all fingers after separate sequential trigger finger releases in a nonrheumatoid hand was reported by Flatt (2007). ${ }^{2}$ He attributed this complication to an "inevitable biomechanical consequence of the sheath-widening liberation," particularly in a multidigit release setting. As the fingers move ulnarwards during the normal grasp, he believed the pulley release incision should "always be placed over the radial side of the sheath" in an attempt to prevent future subluxations. This was thought to preserve the inherent strength of the sheath to resist the ulnar torque of the flexor tendons. ${ }^{2}$

However, there have been no prior reported studies to validate these claims. Although A1 pulleys are commonly incised centrally, whether the location of incision on the A1 pulley has any biomechanical impact on the outcome needs evaluation.

Clear demarcation between the A1 pulley and A2 pulley does not always exist, especially in the presence of inflammation of the tendon sheath and pulley. Conjoint A1 and A2 pulley tendon has also been reported in the literature. ${ }^{4}$ Evaluation and differentiation between the A1 and A2 pulleys through the small incision may add to the challenge, leading to accidental partial or complete A2 pulley release. Knowledge of the safe extent of A2 pulley incision is beneficial.

The aim of this study was to find out if there is any difference between release of A1 pulley radially and ulnarly. As a parallel aim, the safe distal extent of concomitant A2 release was also assessed.

\section{Materials and Methods}

In an experimental cadaveric model, approved by the University of Louisville Willed Body Program, 12 freshly prepared adult cadaveric upper extremity specimens were used. None of the limbs had any prior trauma or surgeries on the hand and forearm. The study was conducted in all digits - a total of 48 digits. The thumb was excluded due to its unique pulley system. In 24 digits ( 6 hands), the A1 pulley was released radially; in the other 24 digits ( 6 hands), the A1 pulley was released ulnarly. Because the null hypothesis stated no difference between the groups and there were no prior similar studies for comparison, a valid power analysis could not be performed. Hence, the sample size was calculated purely based on "resource equation method." ${ }^{6}$ A sample size of 12 hands correlated with adequate E-value of 10 for this study. ( $E$ value $=$ the degree of freedom of analysis of variance [ANOVA], E value of $10-20$ is considered adequate to find difference between the groups). ${ }^{6}$

A longitudinal palmar skin incision was placed over the metacarpophalangeal joint crease. Subcutaneous dissection was performed to identify the A1 and A2 pulleys. Complete release of the A1 pulley was performed by a longitudinal incision radially over the A1 pulley. A tenotomy scissor was passed under the pulley to its most radial or ulnar attachment to the volar plate and the release was completed. Another incision was placed on the volar distal forearm, and then both the flexor digitorum superficialis (FDS) and the flexor digitorum profundus (FDP) tendons were identified. The proximal end of each tendon was sutured to by 2-0 polypropylene suture to a digital pressure gauge. With wrist held in neutral, a traction force of $20 \mathrm{lb}$ was applied manually on the FDS and the FDP (10 lb each), which led to complete flexion of the fingers. Ejeskär et al had demonstrated that the isolated finger flexion force ranged from $13 \mathrm{lb}$ to $23 \mathrm{lb}$ in transverse volar grip. ${ }^{7}$ Hence $20 \mathrm{lb}$ force was selected for the current study.

After application of force to achieve complete finger flexion, the force was released and the fingers were then passively extended. This process was repeated 10 times. The tendons were simultaneously inspected for evidence of tendon subluxation or bowstringing. Bowstringing was defined as the movement of the tendon volar to its normal position during force application. Presence or absence of bowstringing was documented. As bowstringing occurred only during force application with finger in flexed position, the extent of bowstringing could not be measured. Subluxation was defined as movement of the tendon in either a radial or ulnar direction from its original neutral position. Subluxation was measured in millimeters with help of a ruler after the passive extension of the finger.

Subsequently, a graded release of the A2 pulley was performed. Initially $25 \%$ of the A2 pulley was released in line with the A1 incision and application of the force for flexion of the fingers was repeated 10 times. Flexor tendons were watched for presence of bowstringing or amount of subluxation as discussed earlier. These experiments were repeated for individual digits following $50 \%$ and finally $100 \%$ release of the A2 (totally, 96 sets of experiments were performed [-Fig. 1]). Accidental 100\% release of the A2 pulley during trigger finger surgery is unlikely. However, it was still done to check the effect of force vector on tendon subluxation.

For the second group of 24 fingers (6 hands), A1 release was done on ulnar side and the above experiments were repeated in the same fashion (-Fig. 2). During this study a total of 192 sets of experiments were done. Unpaired $t$-test was used to compare the means between the two groups. Social Science Statistics software (https://www.socscistatistics.com/) was used for analysis of the results. $p$ Value $<0.05$ was considered as significant. Results are shown as mean \pm standard deviation (SD).

\section{Results}

\section{Radial vs. Ulnar Release of A1}

There was no bowstringing or subluxation seen after release of the A1 pulley for all the 48 fingers irrespective of whether incision was placed radially or ulnarly. Each tendon takes a different path from wrist to A1 pulley depending on the finger-for the index finger, the tendons go goes radial from the carpal tunnel while for the small finger tendons go in ulnar direction. However, release of A1 had no effect on any of the finger irrespective of the direction taken by its tendon. 

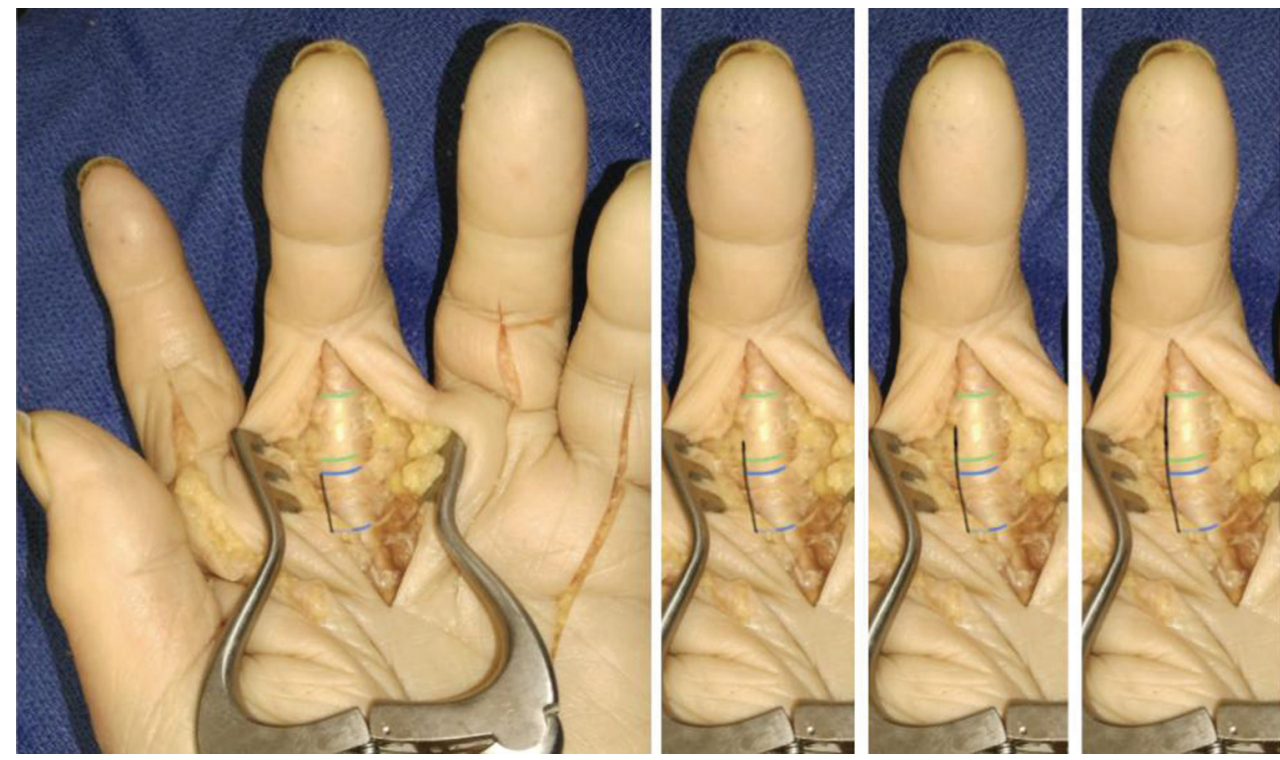

Fig. 1 Schematic diagram showing location of incision for first group of 24 fingers. Initially A1 was released on radial side, followed by serial release of $25 \% A 2,50 \% A 2$, and finally $100 \% A 2$. Blue lines mark the extent of $A 1$ pulley, while green lines mark the extent of $A 2$ pulley. Black lines mark the site of incision. In this specimen there was no clear distinction between $A 1$ and $A 2$ pulleys.
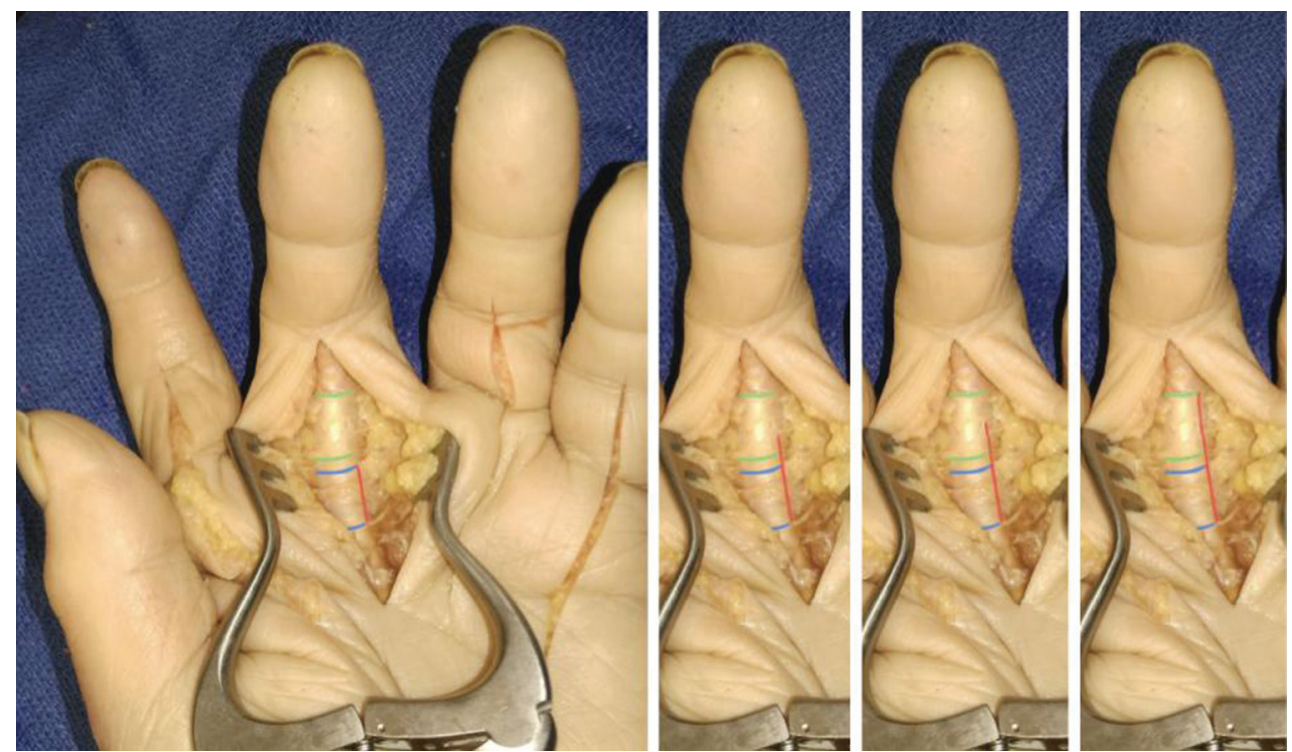

Fig. 2 Schematic diagram showing the location of incision for the second group of 24 fingers on ulnar side. Blue lines mark the extent of A1 pulley, while green lines mark the extent of A2 pulley. Red lines mark the site of incision.

\section{Radial vs. Ulnar Release of A1 + $25 \%$ A2}

Similarly, when the A1 pulley was released along with $25 \%$ of the A2 pulley release, no bowstringing or tendon subluxation was noted in any of the 48 fingers.

\section{Radial vs. Ulnar Release of $A 1+50 \% A 2$}

When the A1 pulley and $50 \%$ of the A2 pulley were released, bowstringing was observed in 3 out of 48 fingers. The bowstringing occurred in one index finger with an ulnar incision, in one small finger with an ulnar incision and in another index finger with a radial incision. However, no subluxation was noted. Therefore, even at 50\% A2 release, there was no significant difference between radial or ulnar release $(0.04 \%$ vs. $0.08 \%, p>0.05$ ).

\section{Radial vs. Ulnar Release of $A 1+100 \% A 2$}

When the A1 pulley and $100 \%$ of the A2 pulley were released, bowstringing occurred immediately in all fingers, and subluxation was observed in 42 out of 48 fingers ( $\boldsymbol{- \text { Fig. }} \mathbf{3}$ ). The subluxation occurred equally from radial (21/24) and ulnar incision (21/24). The average subluxation resulting from radial incision was $2.33 \pm 1.21 \mathrm{~mm}$ for the index fingers, $1.16 \pm 1.63 \mathrm{~mm}$ for the long finger, $1.5 \pm 0.75 \mathrm{~mm}$ for the ring finger, and $1.25 \pm 1.16 \mathrm{~mm}$ for the small finger. The average subluxation for ulnar incision was $3.33 \pm 0.51 \mathrm{~mm}$ for the index finger, $1.66 \pm 1.16 \mathrm{~mm}$ for the long finger, $1.16 \pm$ $0.54 \mathrm{~mm}$ for the ring finger, and $2.16 \pm 1.04 \mathrm{~mm}$ for the small finger ( $\mathbf{- T a b l e ~ 1 )}$ ). The average subluxation was greater for border digits compared with central digits $(2.33 \pm 1.67$ vs. 


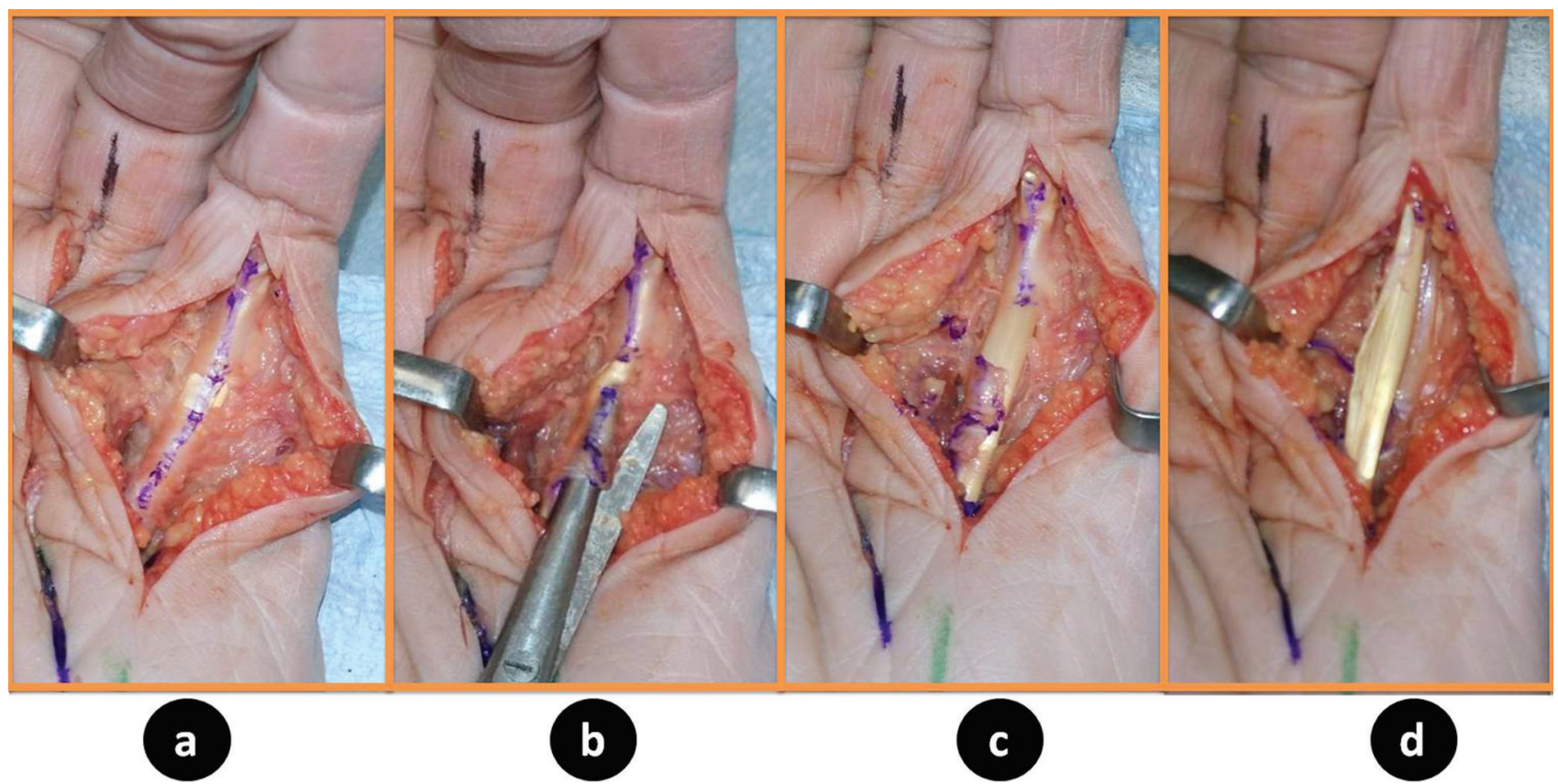

Fig. 3 (a) After opening the skin by vertical incision, the A1 and A2 pulleys are exposed (marked in blue). (b) The A1 pulley being released ulnarly. (c) After complete release of the A1 pulley-no subluxation or bowstringing noted. (d) After complete release of the A2 pulley-bowstringing and radial subluxation noted.

$1.37 \pm 1.05 ; p$ value $=0.002)(-$ Fig. 4$)$. There was no significant statistical difference between radial and ulnar incision groups (1.62 \pm 0.92 vs. $2.08 \pm 1.41 ; p$ value $=0.09)(-$ Table 1$)$.

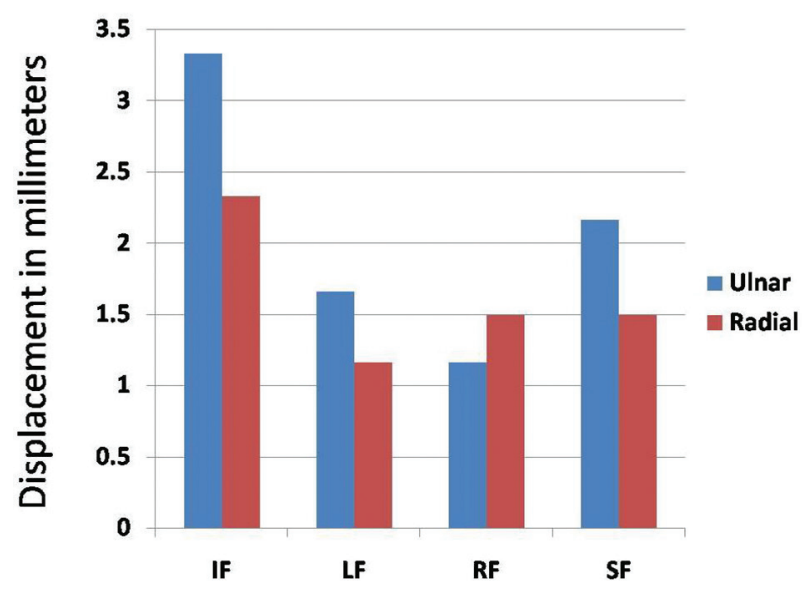

Fig. 4 Bar graph showing the average displacement of radial versus ulnar incision with complete release of the $\mathrm{A} 1$ and $\mathrm{A} 2$ pulleys.

\section{Discussion}

Whether release of the A1 pulley causes any alteration in biomechanical functioning of the hand has been questioned previously by various authors. Peterson et al noted that in primate hands, after the release of the A1 pulley, there was a $0.6 \%$ decrease in tendon excursion and a $10 \%$ increase in work of flexion. These changes were deemed to be minor compared with the release of both $\mathrm{A} 1$ and $\mathrm{A} 2$ pulleys, which resulted in a $20.56 \%$ increase in excursion and a $62.36 \%$ increase in work of flexion. ${ }^{1}$ Using a linear position sensor and a motion capture system, $\mathrm{Lu}$ and colleagues sought to observe any difference between $\mathrm{A} 1$ pulley release alone and $\mathrm{A} 1$ pulley release coupled with release of half of the A2 pulley. ${ }^{8}$ They noted no significant difference in terms of FDP excursion efficiency and moment of the arms of FDS and FDP with respect to the metacarpophalangeal (MCP) joint. They advocated that half of the A2 pulley can be released without significant alteration in tendon biomechanics. ${ }^{8}$ However, they did not study bowstringing or tendon subluxation.

The current study has two significant findings. First, there was no difference between the release of A1 pulley

Table 1 Shows the mean and standard deviation of tendon subluxation in each finger following complete A1 and A2 pulleys

\begin{tabular}{|l|l|l|l|}
\hline Finger & $\begin{array}{l}\text { Ulnar incision } \\
\text { Mean }( \pm \text { SD) }\end{array}$ & $\begin{array}{l}\text { Radial incision } \\
\text { Mean }( \pm \text { SD })\end{array}$ & P value \\
\hline Index finger $(n=12)$ & $3.33( \pm 1.21)$ & $2.33( \pm 0.51)$ & 0.06 \\
\hline Long finger $(n=12)$ & $1.66( \pm 1.63)$ & $1.16( \pm 1.16)$ & 0.24 \\
\hline Ring finger $(n=12)$ & $1.16( \pm 0.75)$ & $1.5( \pm 0.54)$ & 0.25 \\
\hline Small finger $(n=12)$ & $2.16( \pm 1.16)$ & $1.5( \pm 1.04)$ & 0.40 \\
\hline Total $(n=48)$ & $2.08( \pm 1.41)$ & $1.62( \pm 0.92)$ & 0.18 \\
\hline
\end{tabular}


radially or ulnarly. We could not find any previous study which examined the effect of location of A1 release and its impact on tendon subluxation. The current study is the first in this regard and reports that the location of incision does not contribute to bowstringing or subluxation. A. E. Flatt had observed ulnar subluxation following serial release of the A1 pulley in multiple digits and had advised radial release in an attempt to prevent subluxation. ${ }^{2}$ The current study shows that simple biomechanics may not be the reason for such a phenomenon. As discussed below, the cause of subluxation may be inadvertent release of $>50 \%$ A2 or other factors such as inflammation. Since release of A1 pulley radially or ulnarly does not produce any significant difference, release may be done at any location. Due to the proximity of neurovascular bundle to either side of the A1 pulley, the release of the A1 pulley centrally is the safest approach.

Second, no subluxation and bowstringing were encountered when up to $25 \%$ of the A2 pulley was released. Hence, release of up to $25 \%$ of the A2 pulley can be safely done without any concern for bowstringing. This finding is important because during trigger finger release surgery, occasional difficulty is encountered to correctly identify the distal end of the A1 pulley and the beginning of the A2 pulley. This difficulty may be due to either the continuity between the A1 and A2 pulleys, which can be seen in up to $40 \%$ of hands, or the diffuse inflammatory thickening of the flexor sheath. ${ }^{4}$ In such cases, to determine where to stop the pulley release, especially when operating through a limited incision, is a challenge. While incomplete release of A1 can cause relapse of trigger finger, continuation of release through A2 can cause bowstringing. This is a challenge for the surgeon. Currently there are no directions in literature to tackle this issue. The current study attempted to clarify a relationship between the degree of concomitant A2 pulley release along with the A1 pulley and its impact on the bowstringing and subluxation risk.

The current study proposes a safe limit of A2 release. The A2 pulley can be safely released up to $25 \%$ with no consequences. On surface, this corresponds slightly distal to the palmar digital crease. ${ }^{9}$ Hence, in case of difficulty to correctly identify the distal extent of A1, release can be safely done till the level of palmar digital creases. While Lu et al did not see any alteration in tendon biomechanics when $\mathrm{A} 1$ and $50 \% \mathrm{~A} 2$ were released, findings in the current study varied slightly with bowstringing noted in 3 out of 48 fingers (6\%) when $50 \%$ A2 was released.

Bowstringing was first noted at $50 \%$ A2 release (in 6\% of cases); however, subluxation occurred after complete A2 release. Based on these findings, the causative factor for bowstringing and subluxation appears to be the amount of the A2 pulley release rather than the location of incision on A1. Bowstringing has often been listed as a complication of trigger finger release, originally reported by Heithoff et al and widely cited in the literature. ${ }^{10}$ However, the patient reported by Heithoff et al had significant loss of the A2 pulley as well. ${ }^{10}$ The loss of both the $\mathrm{A} 2$ and $\mathrm{A} 1$ pulleys was the probable cause of this bowstringing in that particular patient, and not loss of the A1 pulley alone.
Although finger motions are complex dynamics influenced by multiple tendon forces and multiple joints, the current study attempted a simple design to focus on two factors, the location of A1 release and the extent of concurrent A2 release. The current study proposes practical guideline when difficulty is encountered to clearly delineate the distal limit of A1. Also, the current study provides clarification to previously reported suggestions about the location of A1 release as the cause of subluxation.

The current study is not without its drawbacks. The main drawback of this study is the inability to assess long-term effects. The general imitations of cadaveric model apply to this study. One can obtain only the immediate outcome in a cadaver model. Any factors beyond simple biomechanics cannot be assessed in the current model. Cadaveric findings are not necessarily expected to translate fully in to in vivo results. Second, we did not assess central location of A1 incision. As there was no ulnar subluxation with either radial or ulnar incision, we believe the same would hold true for central incision as well. In addition, whether any of the hands had trigger finger is not known. However, as this study involved release of $\mathrm{A} 1$ and $\mathrm{A} 2$, presence of trigger finger would not have adversely affected the results or interpretation.

\section{Conclusion}

The location of incision for release of the A1 pulley (radial vs. ulnar) has no immediate effect on bowstringing or tendon subluxation in cadaver model. Hence release of the A1 pulley centrally is recommended. In addition, up to $25 \%$ of the A2 pulley can be released safely. When encountered with difficulty to correctly identify the distal margin of A1, safe release can be done up to the level of palmar digital crease. Release of the A1 pulley and $50 \%$ of the A2 pulley can cause bowstringing, while complete release of $\mathrm{A} 1$ and $\mathrm{A} 2$ can lead to bowstringing and subluxation in a cadaver model.

\section{Declaration of Interest}

The authors report no declarations of interest.

\section{Conflict of Interest}

None declared.

\section{Acknowledgments}

The authors would like to thank Dr. Edward A Luce for editorial assistance and Ms. Talicia Tarver for language assistance. The authors wish to also thank the individuals who donated their bodies/tissues for the advancement of education and research at the University of Louisville Willed Body Program.

\section{References}

1 Peterson WW, Manske PR, Bollinger BA, Lesker PA, McCarthy JA. Effect of pulley excision on flexor tendon biomechanics. J Orthop Res 1986;4(1):96-101

2 Flatt AE. Notta's nodules and trigger digits. Proc Bayl Univ Med Cent 2007;20(2):143-145

3 Kozlow JH, Chung KC. Current concepts in the surgical management of rheumatoid and osteoarthritic hands and wrists. Hand Clin 2011;27(1):31-41 
4 Niumsawatt V, Mao D, Salerno S, Rozen WM. Trigger finger release with stepwise preservation of the A1 pulley: a functional pulley-preserving technique. Int Surg 2013;98(4):437-444

5 Lin GT, Cooney WP, Amadio PC, An KN. Mechanical properties of human pulleys. J Hand Surg [Br] 1990;15(4):429-434

6 Charan J, Kantharia ND. How to calculate sample size in animal studies? J Pharmacol Pharmacother 2013;4(4):303-306

7 Ejeskär A, Ortengren R. Isolated finger flexion force-a methodological study. Hand 1981;13(3):223-230
8 Lu SC, Yang TH, Kuo LC, Jou IM, Sun YN, Su FC. Effects of different extents of pulley release on tendon excursion efficiency and tendon moment arms. J Orthop Res 2015;33(2):224-228

9 Wilhelmi BJ, Snyder N IV, VerbeseyJE, Ganchi PA, Lee WP. Trigger finger release with hand surface landmark ratios: an anatomic and clinical study. Plast Reconstr Surg 2001;108(4):908-915

10 Heithoff SJ, Millender LH, Helman J. Bowstringing as a complication of trigger finger release. J Hand Surg Am 1988;13(4):567-570 